\title{
Little Higgs dark matter after PandaX-II/LUX-2016 and LHC Run-1
}

\section{Lei Wu, ${ }^{a, b}$ Bingfang Yang $^{c}$ and Mengchao Zhang ${ }^{d}$}

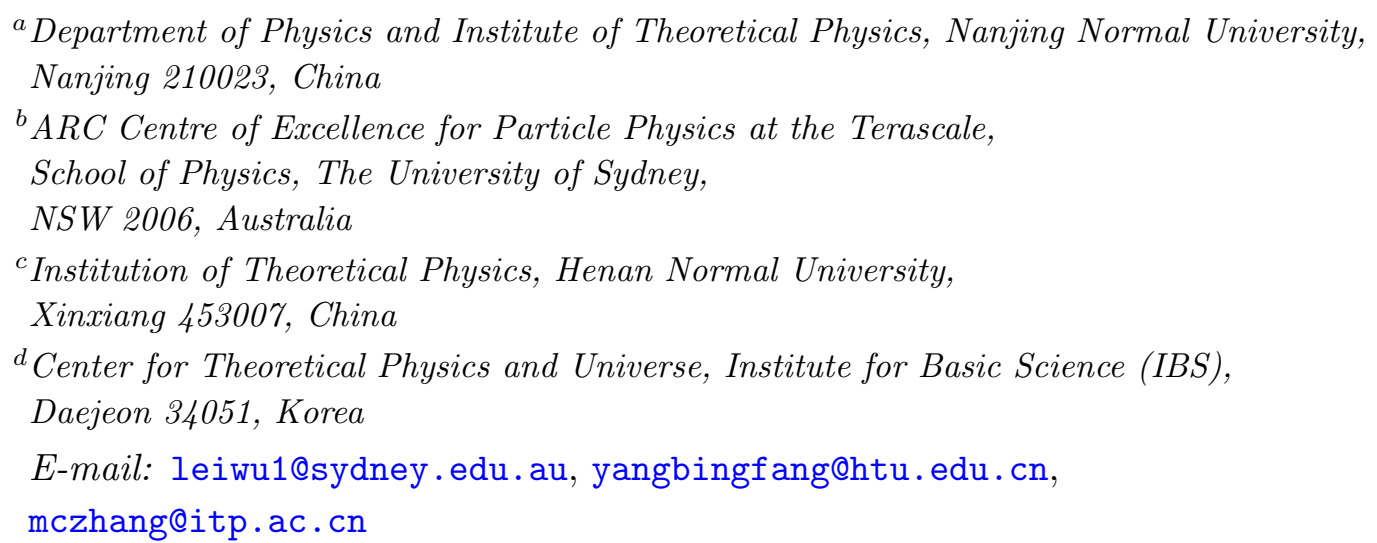

ABSTRACT: In the Littlest Higgs model with $T$-parity (LHT), the $T$-odd heavy photon $\left(A_{H}\right)$ is weakly interacting and can play the role of dark matter. We investigate the lower limit on the mass of $A_{H}$ dark matter under the constraints from Higgs data, EWPOs, $R_{b}$, Planck 2015 dark matter relic abundance, PandaX-II/LUX 2016 direct detections and LHC-8 TeV monojet results. We find that (1) Higgs data, EWPOs and $R_{b}$ can exclude the mass of $A_{H}$ up to $99 \mathrm{GeV}$. To produce the correct dark matter relic abundance, $A_{H}$ has to co-annihilate with $T$-odd quarks $\left(q_{H}\right)$ or leptons $\left(\ell_{H}\right) ;(2)$ the LUX (PandaXII) 2016 data can further exclude $m_{A_{H}}<380(270) \mathrm{GeV}$ for $\ell_{H}-A_{H}$ co-annihilation and $m_{A_{H}}<350(240) \mathrm{GeV}$ for $q_{H}-A_{H}$ co-annihilation; (3) LHC-8 TeV monojet result can give a strong lower limit, $m_{A_{H}}>540 \mathrm{GeV}$, for $q_{H}-A_{H}$ co-annihilation; (4) future XENON1T (2017) experiment can fully cover the parameter space of $\ell_{H}-A_{H}$ co-annihilation and will push the lower limit of $m_{A_{H}}$ up to about $640 \mathrm{GeV}$ for $q_{H^{-}} A_{H}$ co-annihilation.

KEYwords: Phenomenological Models

ARXiv EPrint: 1607.06355 


\section{Contents}

1 Introduction 1

2 Littlest Higgs model with $T$-parity 2

2.1 Heavy photon 2

2.2 T-odd fermions 3

3 Constraints on heavy photon $\boldsymbol{A}_{\boldsymbol{H}}$ dark matter 3

3.1 Higgs data, EWPO and $R_{b} \quad 3$

3.2 Planck relic abundance and PandaX-II/LUX 2016 direct detections 6

3.3 ATLAS-8 TeV monojet limit $\quad 7$

4 Conclusions $\quad 8$

\section{Introduction}

The discovery of $125 \mathrm{GeV}$ Higgs boson $[1,2]$ is a great step towards elucidating the electroweak symmetry breaking mechanism. However, without protection by a symmetry, the Standard Model (SM) Higgs boson mass should be quadratically sensitive to the cutoff scale $\Lambda$ (usually refers to Planck scale $\sim 10^{19} \mathrm{GeV}$ ) via radiative corrections, which renders the theory with $m_{h} \ll \Lambda$ rather unnatural. Besides, the SM cannot explain other convincing observations, such as the dark matter abundance in the Universe. In fact, the electroweak naturalness problem and dark matter are widely considered as major motivations for new physics beyond the SM.

Among various extensions of the SM, the Littlest Higgs model with T-parity (LHT) [3$5]$ is one of the most promising candidates. It can not only successfully solve the electroweak naturalness problem but also provide a viable dark matter candidate. On the theoretical side, the LHT model is based on a non-linear $\sigma$ model describing an $\mathrm{SU}(5) / \mathrm{SO}(5)$ symmetry breaking, which extends the Littlest Higgs model [6-8] by introducing the discrete symmetry $T$-parity. All of the global symmetries that protect the Higgs mass are explicitly broken. The Higgs boson is realized as a pseudo-Nambu-Goldstone boson of the broken global symmetry. With the collective symmetry breaking mechanism, all quadratically divergent contributions to the Higgs mass only first appear at two-loop level, and thus are sufficiently small. On the phenomenological side, the introduction of $T$-parity in the LHT model can relax the electroweak precision observables (EWPOs) bound on the breaking scale $f$ by preventing the tree-level contributions from the heavy gauge bosons [9-15] and lead to an abundant phenomenology at the LHC [16-37]. Besides, the T-parity guarantees that the lightest $T$-odd particle (LTP) is stable so that it can naturally serve as the dark 
matter candidate if it is charge-neutral and colorless. One of such candidates is $T$-odd partner of the hypercharge gauge boson $A_{H} \cdot{ }^{1}$

The phenomenology of heavy photon dark matter has been studied in [40-42]. In general, there are two ways to achieve the correct dark matter relic abundance. One is that two $A_{H}$ dark matter annihilate into SM particles, which is mainly through the $s$ channel via exchanging the Higgs boson. However, due to the constraints of Higgs data and EWPOs, the mass of heavy photon is heavier than $m_{h} / 2$ [43-47]. Thus, without resonant enhancement, the pair annihilation cross section of $A_{H}$ is usually too small to satisfy the observed dark matter relic density. The other is that the $A_{H}$ dark matter co-annihilates with other $T$-odd particles, such as mirror quarks $q_{H}$ or leptons $\ell_{H}$. The co-annihilation of dark matter in simplified models has been studied in [48].

In this work, we will investigate the lower bound on the mass of $A_{H}$ dark matter coannihilations in the LHT model. We will consider various relevant constraints, including Higgs data, EWPOs, $R_{b}$, Planck dark matter relic abundance, PandaX-II/LUX-2016 results and LHC-8 TeV monojet result. This paper is organized as follows. In section 2 , we give a brief description of the heavy photon dark matter and $T$-odd fermion sector of the LHT model. In section 3 , we examine various constraints on $A_{H}$ dark matter. Finally, we draw our conclusions in section 4 .

\section{$2 \quad$ Littlest Higgs model with $T$-parity}

\subsection{Heavy photon}

The LHT model is a realization of non-linear $\sigma$ model, which is based on the coset space $\mathrm{SU}(5) / \mathrm{SO}(5)$. The global symmetry $\mathrm{SU}(5)$ is spontaneously broken into $\mathrm{SO}(5)$ at $\mathrm{TeV}$ scale by the vacuum expectation value (VEV) of the $\Sigma$ field,

$$
\Sigma_{0}=\langle\Sigma\rangle\left(\begin{array}{ccc}
\mathbf{0}_{2 \times 2} & 0 & \mathbf{1}_{2 \times 2} \\
0 & 1 & 0 \\
\mathbf{1}_{2 \times 2} & 0 & \mathbf{0}_{2 \times 2}
\end{array}\right) .
$$

In the meantime, the VEV $\Sigma_{0}$ breaks the gauged subgroup $\left[S U_{1}(2) \times U_{1}(1)\right] \times\left[S U_{2}(2) \times\right.$ $U_{2}(1)$ ] of $\mathrm{SU}(5)$ down to the diagonal $\mathrm{SM}$ electroweak gauge group $S U_{L}(2) \times U_{Y}(1)$. In the end, there are 4 new heavy gauge bosons $W_{H}^{ \pm}, Z_{H}, A_{H}$, whose masses are given at $\mathcal{O}\left(v^{2} / f^{2}\right)$ by

$$
M_{W_{H}}=M_{Z_{H}}=g f\left(1-\frac{v^{2}}{8 f^{2}}\right), \quad M_{A_{H}}=\frac{g^{\prime} f}{\sqrt{5}}\left(1-\frac{5 v^{2}}{8 f^{2}}\right)
$$

where $g$ and $g^{\prime}$ are the SM $S U_{L}(2)$ and $U_{Y}(1)$ gauge couplings, respectively. In order to match the SM prediction for the gauge boson masses, the VEV $v$ needs to be redefined via the functional form

$$
v=\frac{f}{\sqrt{2}} \arccos \left(1-\frac{v_{\mathrm{SM}}^{2}}{f^{2}}\right) \simeq v_{\mathrm{SM}}\left(1+\frac{1}{12} \frac{v_{\mathrm{SM}}^{2}}{f^{2}}\right),
$$

\footnotetext{
${ }^{1}$ Besides $A_{H}, T$-odd partner of neutrino $\nu_{H}$ can be a dark matter candidate as well. However, the direct detection experiments have excluded this possibility because the cross section of elastic scattering between $\nu_{H}$ and nucleus is about $4 \sim 5$ order of magnitude larger than the current experimental bound [39].
} 
where $v_{\mathrm{SM}}=246 \mathrm{GeV}$. The heavy photon $A_{H}$ is typically the lightest $T$-odd state and thus can be a possible candidate for dark matter. The only direct coupling of a pair of $A_{H}$ to the SM sector is via the Higgs boson, resulting in weak-strength cross sections for $A_{H}$ scattering into SM states.

\section{$2.2 \quad T$-odd fermions}

Two fermion SU(2) doublets $q_{1}$ and $q_{2}$ are introduced in the LHT model, where $q_{i}(i=1,2)$ is transformed as a doublet under $\mathrm{SU}(2)_{i}$, and $T$-parity interchanges these two doublets. The $T$-even combination of these two doublets is considered as the $\mathrm{SM} \mathrm{SU}(2)$ doublet, while the $T$-odd combination has to gain a TeV scale mass. The fermion $\mathrm{SU}(2)$ doublets $q_{1}$ and $q_{2}$ are embedded into incomplete SU(5) multiplets $\Psi_{1}$ and $\Psi_{2}$ as $\Psi_{1}=\left(q_{1}, 0,0_{2}\right)^{T}$ and $\Psi_{2}=\left(0_{2}, 0, q_{2}\right)^{T}$, in which $0_{2}=(0,0)^{T}$. Besides, in order to generate masses to the heavy fermions, a $\mathrm{SO}(5)$ multiplet $\Psi_{c}=\left(q_{c}, \chi_{c}, \tilde{q}_{c}\right)^{T}$ is introduced as well. The transformation of $\Psi_{c}$ under the $\mathrm{SU}(5)$ is non-linear: $\Psi_{c} \rightarrow U \Psi_{c}$, where $U$ is the unbroken $\mathrm{SO}(5)$ rotation and is a non-linear representation of the $\mathrm{SU}(5)$. Then, the $T$-invariant Lagrangian for the mass terms of the $T$-odd fermions can be written as follows:

$$
\mathcal{L}_{\kappa}=-\kappa f\left(\bar{\Psi}_{2} \xi \Psi_{c}+\bar{\Psi}_{1} \Sigma_{0} \Omega \xi^{\dagger} \Omega \Psi_{c}\right)+\text { h.c. }
$$

Here $\Omega=\operatorname{diag}(1,1,-1,1,1)$. It should be noted that the non-linear field $\xi$ contains the Higgs field, which can generate the masses of the $T$-odd quarks up to $\mathcal{O}\left(v^{2} / f^{2}\right)$ as,

$$
m_{d_{H}^{i}}=\sqrt{2} \kappa_{d_{i}} f, \quad m_{u_{H}^{i}}=\sqrt{2} \kappa_{u_{i}} f\left(1-\frac{v^{2}}{8 f^{2}}\right)
$$

where $\kappa_{q_{i}}(q=u, d)$ are the diagonalized Yukawa couplings of the $T$-odd quarks. Similarly, the masses of the $T$-odd leptons are given by,

$$
m_{\ell_{H}^{i}}=\sqrt{2} \kappa_{\ell_{i}} f, \quad m_{\nu_{H}^{i}}=\sqrt{2} \kappa_{\nu_{i}} f\left(1-\frac{v^{2}}{8 f^{2}}\right)
$$

where $\kappa_{\ell_{i}}$ and $\kappa_{\nu_{i}}$ are the diagonalized Yukawa couplings of $T$-odd leptons and neutrinos, respectively. From eqs. (2.2), (2.5), (2.6), we note that only if $\kappa_{q_{i}, \ell_{i}, \nu_{i}} \gtrsim 0.11$, the heavy photon $A_{H}$ can become the LTP for a given value of $f$. For simplicity, we assume the universal Yukawa couplings $\kappa_{\ell_{i}}=\kappa_{\nu_{i}}=\kappa_{\ell}$ and $\kappa_{u_{i}}=\kappa_{d_{i}}=\kappa_{q}$, and require the Yukawa couplings $\kappa_{\ell, q}>0.11$.

\section{Constraints on heavy photon $A_{H}$ dark matter}

\subsection{Higgs data, EWPO and $R_{b}$}

In the LHT model, the nature of composite Higgs leads to the deviation of the Higgs gauge couplings from the SM values at the order of $v^{2} / f^{2}$. Moreover, mixing of the SM top with the $T$-even top partner $\left(T^{+}\right)$induces shifts in the Higgs couplings to gluons and photons. 
Here we list the relevant tree-level Higgs couplings for our fitting,

$$
\begin{aligned}
h W^{+} W^{-}: & \frac{2 m_{W}^{2}}{v}\left(1-\frac{1}{6} \frac{v^{2}}{f^{2}}\right) g^{\mu \nu}, & h Z Z: & \frac{2 m_{Z}^{2}}{v}\left(1-\frac{1}{6} \frac{v^{2}}{f^{2}}\right) g^{\mu \nu}, \\
h t \bar{t}: & -\frac{m_{t}}{v}\left[1+\frac{v^{2}}{f^{2}}\left(-\frac{2}{3}+\frac{R^{2}}{\left(1+R^{2}\right)^{2}}\right)\right], & h T^{+} \bar{T}^{-}: & \frac{m_{T}}{v} \frac{R^{2}}{\left(1+R^{2}\right)^{2}} \frac{v^{2}}{f^{2}},
\end{aligned}
$$

where $R$ is the mixing angle between the top quark and $T^{+}$quark. The loop-induced couplings $h g g$ and $h \gamma \gamma$ are given in [18]. Besides, there are two possible ways to construct $T$-invariant Lagrangians of the Yukawa interactions of the charged leptons and down-type quarks. Up to $\mathcal{O}\left(v_{\mathrm{SM}}^{4} / f^{4}\right)$, the ratios of the down-type quark Yukawa couplings $g_{h d \bar{d}}$ with respect to the SM prediction are given by [18],

$$
\begin{array}{ll}
\frac{g_{h \bar{d} d}}{g_{h \bar{d} d}^{\mathrm{SM}}}=1-\frac{1}{4} \frac{v_{\mathrm{SM}}^{2}}{f^{2}}+\frac{7}{32} \frac{v_{\mathrm{SM}}^{4}}{f^{4}} & \text { Case A } \\
\frac{g_{h \bar{d} d}}{g_{h \bar{d} d}^{\mathrm{SM}}}=1-\frac{5}{4} \frac{v_{\mathrm{SM}}^{2}}{f^{2}}-\frac{17}{32} \frac{v_{\mathrm{SM}}^{4}}{f^{4}} & \text { Case B. }
\end{array}
$$

In our following fitting, we will confront the above modified Higgs couplings $h V V, h f \bar{f}$, $h g g$ and $h \gamma \gamma$ with the Higgs data for both cases.

In the LHT model, the electroweak precision observables $S$ and $T$ are changed by the modified Higgs gauge couplings $h V V$ [15]. Furthermore, the top partner can correct the propagators of the electroweak gauge bosons at one-loop level. The UV operators can also contribute to the $S$ and $T$ parameters [49]. We take the couplings of the UV operators as unity [43]. Besides, the new mirror fermions and new gauge bosons can contribute to the $Z b \bar{b}$ coupling at one-loop level [51-53]. We will include the EWPOs and $R_{b}$ constraints in our study.

On the other hand, the current LHC direct searches for the multi-jet with the transverse missing energy can also produce the bounds on the parameter space of the LHT model. However, they are not strong enough to push the exclusion limits much beyond the indirect constraints [43]. In particular, the ATLAS and CMS collaborations performed the searches for the vector-like top partner in different final states $b W, t Z$ and $t h$. During the LHC Run1, they excluded the masses of the top partners up to about $700 \mathrm{GeV}$ [54-56]. However, those bounds depend on the assumptions of the group representations of top partners and their decay channels. In addition to the conventional decay channels ( $b W, t Z$ and $t h)$, the $T$-even top partner $T^{+}$can decay to $T^{-} A_{H}$, which can weaken the current LHC bounds on top partner in the LHT model [36]. So in our scan, we consider the indirect constraints including Higgs data, EWPOs and $R_{b}$.

We scan over the free parameters $\kappa, f$ and $R$ within the following ranges,

$$
500 \mathrm{GeV}<f<5000 \mathrm{GeV}, \quad 0.11<\kappa<0.2, \quad 0.1<R<3.3 .
$$

where we assume $\kappa_{\ell}=\kappa_{q}=\kappa$. In order to escape LHC limits from the multijet with $Z_{T}$, we require $\kappa \leqslant 0.2$ to forbidden $T$-odd fermions decay to the heavy gauge bosons $Z_{H}$ and $W_{H}$. Besides, we decouple the $T$-odd top quark $t_{-}$by setting $m_{t_{-}}=3 \mathrm{TeV}$ in order to 

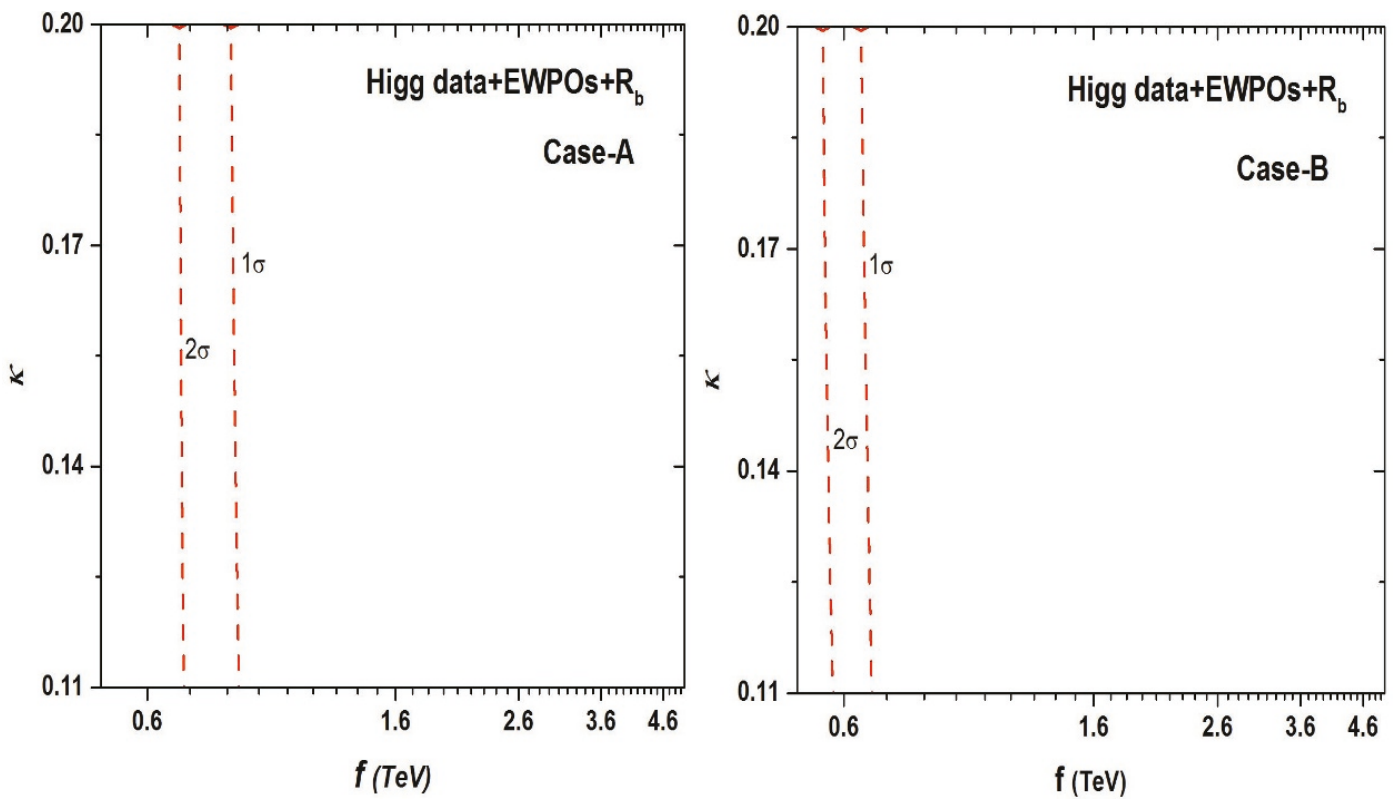

Figure 1. Excluded regions (left each contour) in the plane of $\kappa$ versus $f$ for Case A and Case B, where the parameter $R$ is marginalized over.

avoid the bound of LHC searches for long-lived charged particles. We adopt our previous scan method $[45,46]$ by constructing the likelihood $\mathcal{L} \equiv \exp \left[-\sum \chi_{i}^{2}\right]$ for each point, where index $i$ denotes the following constraint:

(1) The electroweak precision observables: $S, T$ and $U$ [15]. We use the experimental values of $S, T$ and $U$ from ref. [50].

(2) $R_{b}$ [52]. We use the final combined result $R_{b}=0.21629 \pm 0.00066$ [50] from the LEP and SLD measurements.

(3) Higgs data. We check the LHT Higgs couplings by using HiggsSignals-1.4.0 [57, 58], which includes the available Higgs data sets from the ATLAS, CMS, CDF and D0 collaborations. The mass-centered $\chi^{2}$ method is chosen in our study.

On the other hand, since the SM flavor symmetry is broken by the extension of the top quark sector, the mixing between top partner and down-type quark can induce flavor changing neutral current processes at one-loop level [59-62]. We checked our samples and found that the constraints from $B_{s} \rightarrow \mu^{+} \mu^{-}[63,64]$ can be easily satisfied within the current uncertainty.

In figure 1, we show the excluded regions (left each contour) in the plane of $\kappa$ versus $f$ for Case A and Case B by fitting Higgs data, EWPOs and $R_{b}$. The parameter $R$ is marginalized over. From the figure 1, it can be seen that the lower bound on the symmetry breaking scale $f$ is almost independent of $\kappa$ and has reached about 675 (550) GeV at $2 \sigma$ level in Case A (B), which corresponds to $m_{A_{H}}=99(76) \mathrm{GeV}$. Since the reduced bottom Yukawa coupling in Case B is smaller than that in Case A (cf. eq. (3.2)), the non-fermionic 

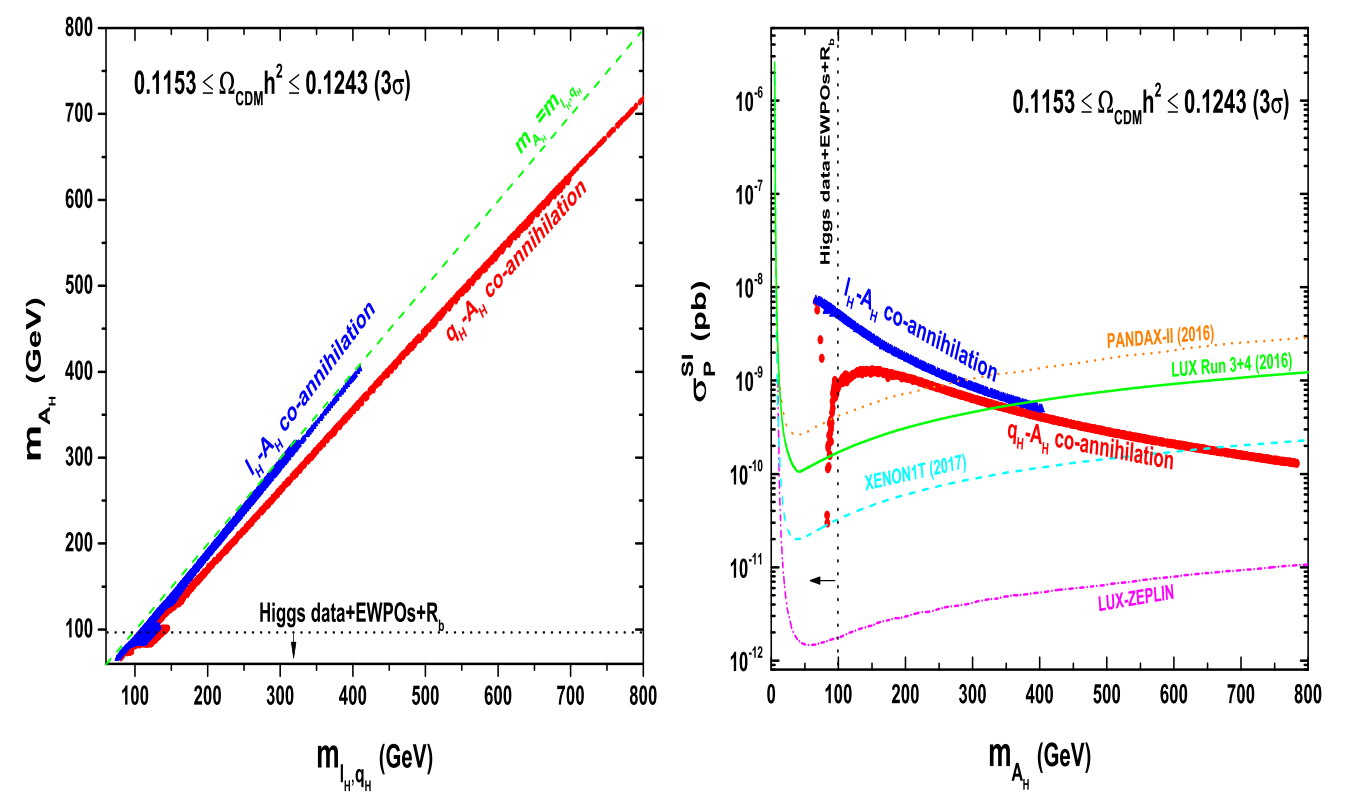

Figure 2. The scatter plot on the planes of $m_{A_{H}}$ versus $m_{\ell_{H}, q_{H}}$ (left panel) and $\sigma_{p}^{\text {SI }}$ versus $m_{A_{H}}$ (right panel). All samples satisfy the Planck dark matter relic abundance within $3 \sigma$ range. For $\ell_{H}-A_{H}\left(q_{H}-A_{H}\right)$ co-annihilation, $\kappa_{q}\left(\kappa_{\ell}\right)$ is fixed at 3.

decays of the Higgs boson can be enhanced in Case B, which is more consistent with the current ATLAS-8 TeV Higgs data. So the lower bound on $f$ in Case B is weaker than that in Case A. To conservatively examine dark matter and LHC experiment constraints on heavy photon $A_{H}$, we will focus on Case $\mathrm{A}$ in the following.

\subsection{Planck relic abundance and PandaX-II/LUX 2016 direct detections}

In the LHT, $A_{H}$ pair mainly annihilates through a Higgs boson in $s$-channel to a pair of fermions, $W / Z$ bosons and Higgs bosons, whose contributions to the relic density depend on the mass of $A_{H}$. When $m_{A_{H}}$ is heavier than $m_{h} / 2$, the Higgs resonance effect in $A_{H}$ pair annihilation will become small and the $A_{H} A_{H}$ annihilation cross section will be too small to give the right relic abundance. We use the MicrOMEGAs4.2.5 [65] to calculate the relic density $\Omega h^{2}$ and the spin-independent scattering cross section between DM and nucleon $\sigma_{p}^{\mathrm{SI}}$.

In the left panel of figure 2, we show the scatter plot on the plane of $m_{A_{H}}$ versus $m_{\ell_{H}, q_{H}}$. We require samples to satisfy the Planck dark matter relic abundance within $3 \sigma$ range. We can see that the constraint of the relic density requires $A_{H}$ co-annihilate with $T$-odd fermions, which is typically given by,

$$
\begin{aligned}
\frac{\Delta m_{\ell_{H}}}{m_{A_{H}}} & =\frac{m_{\ell_{H}}-m_{A_{H}}}{m_{A_{H}}} \lesssim 0.1 \\
\frac{\Delta m_{q_{H}}}{m_{A_{H}}} & =\frac{m_{q_{H}}-m_{A_{H}}}{m_{A_{H}}} \lesssim 0.2
\end{aligned}
$$


In the calculation of co-annihilation, the effective dark matter annihilation cross section $\sigma_{\text {eff }}\left(A_{H}\right)$ includes the contributions from $A_{H}$ pair annihilation, $A_{H}$ and $\ell_{H} / q_{H}$ coannihilation and $\ell_{H} / q_{H}$ pair annihilation [66]. For the colored co-annihilation partner $q_{H}$, the contribution of $q_{H}$ pair annihilation is large because of the strong coupling. While for the non-colored co-annihilation partner $\ell_{H}$, three contributions are comparable. So the annihilation cross section of $A_{H}-\ell_{H}$ is smaller than that of $A_{H}-q_{H}$ for the given mass splitting [48]. To obtain the correct relic density, the mass splitting between $A_{H}$ and co-annihilation partner in $A_{H}-\ell_{H}$ co-annihilation has to be smaller than that in $A_{H}-\ell_{q}$ co-annihilation (cf. eq. (3.5)). When $A_{H}$ becomes heavy, the effective cross section $\sigma_{\text {eff }}\left(A_{H}\right)$ decreases so that the dark matter relic density will be overproduced in the universe. This leads to the upper bounds on the masses of dark matter and its co-annihilation partners. Due to the small co-annihilation cross section, the resulting viable region of parameter space for the dark matter relic density only extends to about $400 \mathrm{GeV}$ in $A_{H}-\ell_{H}$ co-annihilation.

In the right panel of figure 2 , we show the scatter plot on the plane of $\sigma_{p}^{\text {SI }}$ versus $m_{A_{H}}$. There are three processes contributing to the cross-section of $A_{H}$ scattering off nucleon: Higgs-boson-exchanged $t$-channel, $T$-odd-quark-exchanged $t$-channel and $s$-channel [40]. For $\ell_{H}-A_{H}$ co-annihilation, the dominant contribution to $\sigma_{p}^{\mathrm{SI}}$ is the Higgs-boson-exchanged $t$-channel since the $T$-odd quarks are decoupled. The mass of $A_{H}$ can be excluded up to about 380 (270) GeV by the LUX (PandaX-II) 2016 data $[67,68]$. While for $q_{H}-A_{H}$ co-annihilation, $m_{A_{H}}<350(240) \mathrm{GeV}$ is excluded by the LUX (PandaX-II) 2016 data. This is because that the cancellation between $T$-odd quark and the top quark loops in $h g g$ coupling reduces the contribution of Higgs-boson-exchanged $t$-channel to cross section $\sigma_{p}^{\mathrm{SI}}$. Besides, the amplitudes of $T$-odd-quark-exchanged $t$-channel and $s$-channel interference destructively in our parameter space. The expected XENON1T (2017) experiment [69] will allow it to cover $\ell_{H}-A_{H}$ co-annihilation region and push the lower limit of $m_{A_{H}}$ up to $640 \mathrm{GeV}$.

\subsection{ATLAS-8 TeV monojet limit}

In co-annihilations, the decay products of light $T$-odd lepton or quark are usually very soft. One way of probing such a compressed region is to use the ISR/FSI jet to boost the soft objects, which produces the monojet(-like) events at the LHC [70-72]. Since the $T$-odd leptons are produced via the electroweak interaction, the cross section of the heavy lepton pair production are much smaller than that of the heavy quark pair production at the LHC. We checked and found that the ATLAS monojet data can not give an exclusion limit on the $\ell_{H}-A_{H}$ co-annihilation scenario. So we only present the results for $q_{H}-A_{H}$ co-annihilation in our work.

We recast the ATLAS-8 TeV monojet bound [73] by using CheckMATE-1.2.1 [74, 75]. In our scenario, the monojet events arise from the processes:

$$
p p \rightarrow j q_{H} A_{H}, \quad j q_{H} \bar{q}_{H}
$$

We generate the parton level signal events by using MadGraph5_aMC@NLO [76]. Then, the parton level events are showered and hadronized by PYTHIA [77]. The fast detector 

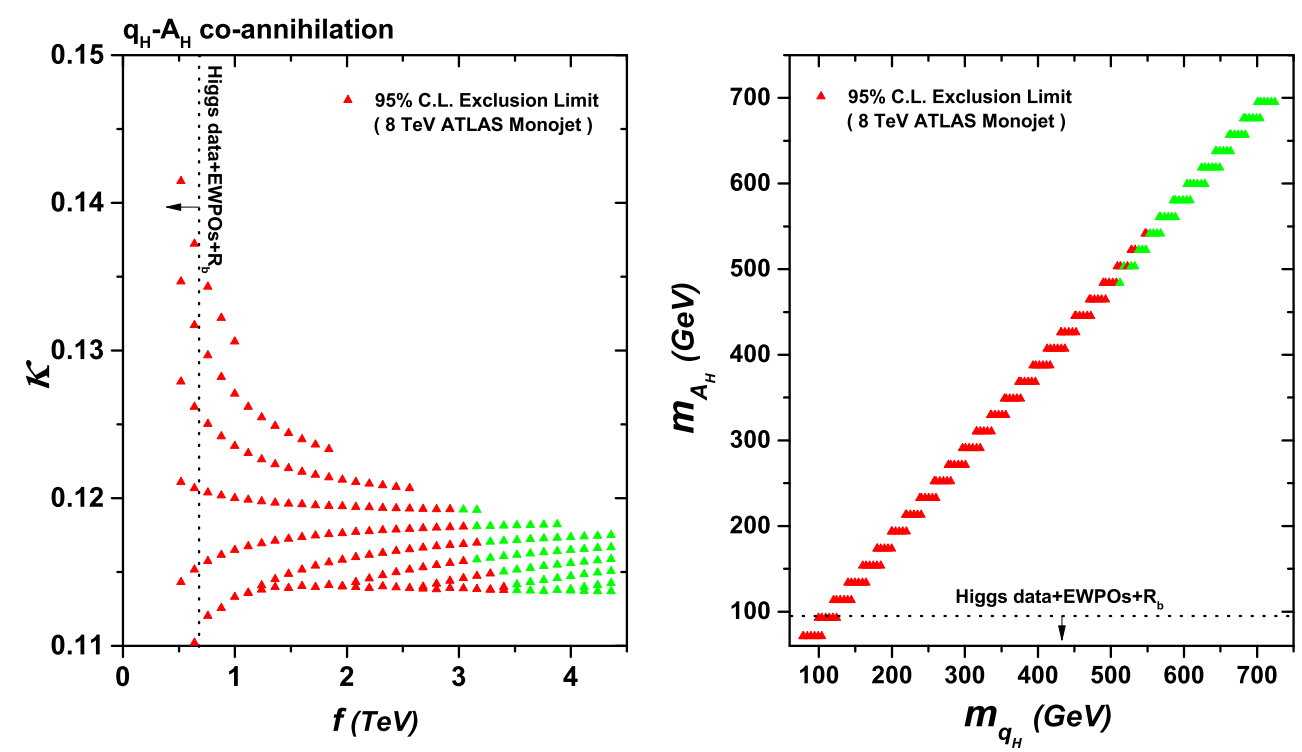

Figure 3. Same as figure 2, but for ATLAS-8 TeV monojet constraint on $q_{H}-A_{H}$ co-annihilation.

simulation are performed with the tuned Delphes [78]. The jet is clustered by FastJet [79] with the anti- $k_{t}$ algorithm [80]. We normalize the cross section of $q_{H} \bar{q}_{H}$ and $q_{H} A_{H}$ productions to their NLO value by including a $K$-factor 1.5 [81]. Finally, we define the ratio $r=\max \left(N_{S, i} / S_{\mathrm{obs}, i}^{95 \%}\right)$ to estimate the exclusion limit. Here $N_{S, i}$ is the event number of signal for $i$-th signal region and $S_{\mathrm{obs}, i}^{95 \%}$ is the corresponding observed $95 \%$ C.L. upper limit. The max is over all signal regions in the analysis. We conclude that a point is excluded at $95 \%$ C.L. if $r>1$. In figure 3, we show the monojet constraints on the parameter space of $q_{H}-A_{H}$ co-annihilation. We can see that the monojet limit can exclude the scale $f$ up to $3.4 \mathrm{TeV}$, which corresponds to $m_{A_{H}}>540 \mathrm{GeV}$. For a given $f$, the monojet has a better sensitivity in the region with small Yukawa coupling $\kappa_{q}$.

\section{Conclusions}

In this work, we investigate the lower limit on the mass of $A_{H}$ dark matter by using the constraints from Higgs data, EWPOs, $R_{b}$, Planck dark matter relic abundance, LUX direct detection and LHC-8 TeV monojet results. We find that the mass of $A_{H}$ has been excluded up to $99 \mathrm{GeV}$ by Higgs data, EWPOs and $R_{b}$. Therefore, $A_{H}$ needs to co-annihilate with $T$ odd quarks $\left(q_{H}\right)$ or leptons $\left(\ell_{H}\right)$ to give the correct dark matter relic abundance. Further, with the very recent LUX (PandaX-II) 2016 data, the lower limit of $m_{A_{H}}$ can be pushed up to about $380(270) \mathrm{GeV}$ and $350(240) \mathrm{GeV}$ for $\ell_{H}-A_{H}$ and $q_{H}-A_{H}$ co-annihilations, respectively. Also, we find that ATLAS $8 \mathrm{TeV}$ monojet result can give a stringent lower limit, $m_{A_{H}}>540 \mathrm{GeV}$, for $q_{H}-A_{H}$ co-annihilation, while can not produce the limit on $m_{A_{H}}$ for $l_{H}-A_{H}$ co-annihilation. In future XENON1T (2017) experiment, parameter space of $\ell_{H}-A_{H}$ co-annihilation can be fully covered and the lower limit of $m_{A_{H}}$ will be pushed up to about $640 \mathrm{GeV}$ for $q_{H}-A_{H}$ co-annihilation. 


\section{Acknowledgments}

Lei Wu thanks the helpful discussions with Dr. Lei Wang. This work is partly supported by the Australian Research Council, by the National Natural Science Foundation of China (NNSFC) under grants Nos. 11275057, 11305049, 11375001 and 11405047, by Specialised Research Fund for the Doctoral Program of Higher Education under Grant No. 20134104120002 and by the Startup Foundation for Doctors of Henan Normal University under contract No. 11112, by the China Postdoctoral Science Foundation under Grant No. 2014M561987 and the Joint Funds of the National Natural Science Foundation of China (U1404113).

Open Access. This article is distributed under the terms of the Creative Commons Attribution License (CC-BY 4.0), which permits any use, distribution and reproduction in any medium, provided the original author(s) and source are credited.

\section{References}

[1] ATLAS collaboration, Observation of a new particle in the search for the Standard Model Higgs boson with the ATLAS detector at the LHC, Phys. Lett. B 716 (2012) 1 [arXiv: 1207.7214] [INSPIRE].

[2] CMS collaboration, Observation of a new boson at a mass of $125 \mathrm{GeV}$ with the CMS experiment at the LHC, Phys. Lett. B 716 (2012) 30 [arXiv:1207.7235] [INSPIRE].

[3] H.-C. Cheng and I. Low, TeV symmetry and the little hierarchy problem, JHEP 09 (2003) 051 [hep-ph/0308199] [INSPIRE].

[4] H.-C. Cheng and I. Low, Little hierarchy, little Higgses and a little symmetry, JHEP 08 (2004) 061 [hep-ph/0405243] [INSPIRE].

[5] I. Low, T-parity and the littlest Higgs, JHEP 10 (2004) 067 [hep-ph/0409025] [INSPIRE].

[6] N. Arkani-Hamed, A.G. Cohen and H. Georgi, Electroweak symmetry breaking from dimensional deconstruction, Phys. Lett. B 513 (2001) 232 [hep-ph/0105239] [INSPIRE].

[7] N. Arkani-Hamed, A.G. Cohen, E. Katz, A.E. Nelson, T. Gregoire and J.G. Wacker, The Minimal moose for a little Higgs, JHEP 08 (2002) 021 [hep-ph/0206020] [INSPIRE].

[8] N. Arkani-Hamed, A.G. Cohen, E. Katz and A.E. Nelson, The Littlest Higgs, JHEP 07 (2002) 034 [hep-ph/0206021] [INSPIRE].

[9] C. Csáki, J. Hubisz, G.D. Kribs, P. Meade and J. Terning, Big corrections from a little Higgs, Phys. Rev. D 67 (2003) 115002 [hep-ph/0211124] [InSPIRE].

[10] C. Csáki, J. Hubisz, G.D. Kribs, P. Meade and J. Terning, Variations of little Higgs models and their electroweak constraints, Phys. Rev. D 68 (2003) 035009 [hep-ph/0303236] [INSPIRE].

[11] J.L. Hewett, F.J. Petriello and T.G. Rizzo, Constraining the littlest Higgs, JHEP 10 (2003) 062 [hep-ph/0211218] [INSPIRE].

[12] M.-C. Chen and S. Dawson, One loop radiative corrections to the $\rho$ parameter in the littlest Higgs model, Phys. Rev. D 70 (2004) 015003 [hep-ph/0311032] [INSPIRE]. 
[13] W. Kilian and J. Reuter, The Low-energy structure of little Higgs models, Phys. Rev. D 70 (2004) 015004 [hep-ph/0311095] [INSPIRE].

[14] G. Marandella, C. Schappacher and A. Strumia, Little-Higgs corrections to precision data after LEP2, Phys. Rev. D 72 (2005) 035014 [hep-ph/0502096] [INSPIRE].

[15] J. Hubisz, P. Meade, A. Noble and M. Perelstein, Electroweak precision constraints on the littlest Higgs model with T-parity, JHEP 01 (2006) 135 [hep-ph/0506042] [INSPIRE].

[16] T. Han, H.E. Logan, B. McElrath and L.-T. Wang, Phenomenology of the little Higgs model, Phys. Rev. D 67 (2003) 095004 [hep-ph/0301040] [INSPIRE].

[17] J. Hubisz and P. Meade, Phenomenology of the littlest Higgs with T-parity, Phys. Rev. D 71 (2005) 035016 [hep-ph/0411264] [INSPIRE].

[18] C.-R. Chen, K. Tobe and C.P. Yuan, Higgs boson production and decay in little Higgs models with T-parity, Phys. Lett. B 640 (2006) 263 [hep-ph/0602211] [INSPIRE].

[19] H.-C. Cheng, I. Low and L.-T. Wang, Top partners in little Higgs theories with T-parity, Phys. Rev. D 74 (2006) 055001 [hep-ph/0510225] [INSPIRE].

[20] A. Freitas and D. Wyler, Phenomenology of mirror fermions in the littlest Higgs model with T-parity, JHEP 11 (2006) 061 [hep-ph/0609103] [INSPIRE].

[21] A. Belyaev, C.-R. Chen, K. Tobe and C.P. Yuan, Phenomenology of littlest Higgs model with $T^{-}$-parity: including effects of $T^{-}$odd fermions, Phys. Rev. D 74 (2006) 115020 [hep-ph/0609179] [INSPIRE].

[22] D. Choudhury and D.K. Ghosh, LHC signals of T-odd heavy quarks in the Littlest Higgs model, JHEP 08 (2007) 084 [hep-ph/0612299] [INSPIRE].

[23] Q.-H. Cao and C.-R. Chen, Signatures of extra gauge bosons in the littlest Higgs model with T-parity at future colliders, Phys. Rev. D 76 (2007) 075007 [arXiv:0707.0877] [InSPIRE].

[24] S. Matsumoto, M.M. Nojiri and D. Nomura, Hunting for the Top Partner in the Littlest Higgs Model with T-parity at the CERN LHC, Phys. Rev. D 75 (2007) 055006 [hep-ph/0612249] [INSPIRE].

[25] Q.-H. Cao, C.S. Li and C.P. Yuan, Impact of Single-Top Measurement to Littlest Higgs Model with T-Parity, Phys. Lett. B 668 (2008) 24 [hep-ph/0612243] [INSPIRE].

[26] L. Wang and J.M. Yang, Higgs boson productions at LHC as a probe of different littlest Higgs models with T-parity, Phys. Rev. D 77 (2008) 015020 [arXiv:0710.5038] [INSPIRE].

[27] Q.-H. Cao, C.-R. Chen, F. Larios and C.P. Yuan, Anomalous gt $\bar{t}$ couplings in the Littlest Higgs Model with T-parity, Phys. Rev. D 79 (2009) 015004 [arXiv:0801.2998] [INSPIRE].

[28] J.-Y. Liu, Z.-G. Si and C.-X. Yue, Littlest Higgs model with T parity and top quark pair production at LHC, Phys. Rev. D 81 (2010) 015011 [INSPIRE].

[29] S. Matsumoto, T. Moroi and K. Tobe, Testing the Littlest Higgs Model with T parity at the Large Hadron Collider, Phys. Rev. D 78 (2008) 055018 [arXiv: 0806.3837] [INSPIRE].

[30] S. Yang, Associated production of the Higgs boson and a single top quark in the littlest Higgs model at Large Hadron Collier, Phys. Lett. B 675 (2009) 352 [arXiv:0904.1646] [INSPIRE].

[31] D. Choudhury, D.K. Ghosh and S.K. Rai, Dijet Signals of the Little Higgs Model with T Parity, JHEP 07 (2012) 013 [arXiv: 1202.4213] [INSPIRE]. 
[32] R.-Y. Zhang, H. Yan, W.-G. Ma, S.-M. Wang, L. Guo and L. Han, $W_{H} / Z_{H}$ production associated with a T-odd (anti)quark at the LHC in NLO QCD, Phys. Rev. D 85 (2012) 015017 [arXiv: 1112.5225] [INSPIRE].

[33] S.-J. Xiong, W.-G. Ma, L. Guo, C. Chen and R.-Y. Zhang, NLO QCD corrections to same-sign T-odd quark pair production in the littlest Higgs model with T-parity at the LHC, Phys. Rev. D 89 (2014) 114015 [arXiv: 1406. 0584] [InSPIRE].

[34] L.-W. Chen, R.-Y. Zhang, W.-G. Ma, W.-H. Li, P.-F. Duan and L. Guo, Probing the littlest Higgs model with $T$ parity using di-Higgs events through $Z_{H}$-pair production at the LHC in NLO QCD, Phys. Rev. D 90 (2014) 054020 [arXiv: 1409.1338] [INSPIRE].

[35] B. Yang, J. Han and N. Liu, Associated production of single top and Higgs at the LHC in the littlest Higgs model with T parity, JHEP 04 (2015) 148 [arXiv:1412.2927] [INSPIRE].

[36] N. Liu, L. Wu, B. Yang and M. Zhang, Single top partner production in the Higgs to diphoton channel in the Littlest Higgs Model with T parity, Phys. Lett. B 753 (2016) 664 [arXiv: 1508.07116] [INSPIRE].

[37] Q.-H. Cao, C.-R. Chen, Y. Liu and Y. Liu, Testing the littlest Higgs model with T parity at the LHC Run-II, Phys. Rev. D 94 (2016) 055033 [arXiv:1512.09144] [InSPIRE].

[38] D. Choudhury, D.K. Ghosh, S.K. Rai and I. Saha, Little Higgs after the little one, JHEP 06 (2016) 074 [arXiv : 1603.02037] [INSPIRE].

[39] G. Servant and T.M.P. Tait, Elastic scattering and direct detection of Kaluza-Klein dark matter, New J. Phys. 4 (2002) 99 [hep-ph/0209262] [INSPIRE].

[40] A. Birkedal, A. Noble, M. Perelstein and A. Spray, Little Higgs dark matter, Phys. Rev. D 74 (2006) 035002 [hep-ph/0603077] [INSPIRE].

[41] L. Wang, J.M. Yang and J. Zhu, Dark matter in the little Higgs model under current experimental constraints from the LHC, Planck and Xenon data, Phys. Rev. D 88 (2013) 075018 [arXiv: 1307.7780] [INSPIRE].

[42] C.-R. Chen, M.-C. Lee and H.-C. Tsai, Implications of the Little Higgs Dark Matter and T-odd Fermions, JHEP 06 (2014) 074 [arXiv: 1402.6815] [INSPIRE].

[43] J. Reuter, M. Tonini and M. de Vries, Littlest Higgs with T parity: Status and Prospects, JHEP 02 (2014) 053 [arXiv: 1310.2918] [INSPIRE].

[44] J. Reuter and M. Tonini, Can the 125 GeV Higgs be the Little Higgs?, JHEP 02 (2013) 077 [arXiv: 1212.5930] [INSPIRE].

[45] B. Yang, G. Mi and N. Liu, Higgs couplings and Naturalness in the littlest Higgs model with $T$ parity at the LHC and TLEP, JHEP 10 (2014) 047 [arXiv: 1407.6123] [INSPIRE].

[46] C. Han, A. Kobakhidze, N. Liu, L. Wu and B. Yang, Constraining Top partner and Naturalness at the LHC and TLEP, Nucl. Phys. B 890 (2014) 388 [arXiv:1405.1498] [INSPIRE].

[47] X.-F. Han, L. Wang, J.M. Yang and J. Zhu, Little Higgs theory confronted with the LHC Higgs data, Phys. Rev. D 87 (2013) 055004 [arXiv: 1301.0090] [INSPIRE].

[48] M.J. Baker et al., The Coannihilation Codex, JHEP 12 (2015) 120 [arXiv:1510.03434] [INSPIRE].

[49] Z. Han and W. Skiba, Effective theory analysis of precision electroweak data, Phys. Rev. D 71 (2005) 075009 [hep-ph/0412166] [INSPIRE]. 
[50] Particle Data Group collaboration, K.A. Olive et al., Review of Particle Physics, Chin. Phys. C 38 (2014) 090001 [InSPIRE].

[51] C.-x. Yue and W. Wang, The Branching ratio $R_{b}$ in the littlest Higgs model, Nucl. Phys. B 683 (2004) 48 [hep-ph/0401214] [INSPIRE].

[52] X.-F. Han, $R_{b}$ Constraints on Littlest Higgs Model with T parity, Phys. Rev. D 80 (2009) 055027 [arXiv: 0908.2572] [inSPIRE].

[53] B. Yang, X. Wang and J. Han, The Study of the contribution of the LHT model to Z $b \bar{b}$ coupling, Nucl. Phys. B 847 (2011) 1 [arXiv:1103.2506] [INSPIRE].

[54] ATLAS collaboration, Search for pair and single production of new heavy quarks that decay to a $Z$ boson and a third-generation quark in pp collisions at $\sqrt{s}=8 \mathrm{TeV}$ with the ATLAS detector, JHEP 11 (2014) 104 [arXiv:1409.5500] [INSPIRE].

[55] ATLAS collaboration, Search for production of vector-like quark pairs and of four top quarks in the lepton plus jets final state in pp collisions at $\sqrt{s}=8 \mathrm{TeV}$ with the ATLAS detector, ATLAS-CONF-2015-012, ATLAS-COM-CONF-2015-012 (2015).

[56] CMS collaboration, Inclusive search for a vector-like T quark with charge $\frac{2}{3}$ in pp collisions at $\sqrt{s}=8 \mathrm{TeV}$, Phys. Lett. B 729 (2014) 149 [arXiv:1311.7667] [INSPIRE].

[57] P. Bechtle, S. Heinemeyer, O. Stål, T. Stefaniak and G. Weiglein, HiggsSignals: Confronting arbitrary Higgs sectors with measurements at the Tevatron and the LHC, Eur. Phys. J. C $\mathbf{7 4}$ (2014) 2711 [arXiv: 1305.1933] [INSPIRE].

[58] P. Bechtle, O. Brein, S. Heinemeyer, G. Weiglein and K.E. Williams, HiggsBounds: Confronting Arbitrary Higgs Sectors with Exclusion Bounds from LEP and the Tevatron, Comput. Phys. Commun. 181 (2010) 138 [arXiv:0811.4169] [INSPIRE].

[59] J. Hubisz, S.J. Lee and G. Paz, The Flavor of a little Higgs with T parity, JHEP 06 (2006) 041 [hep-ph/0512169] [INSPIRE].

[60] M. Blanke, A.J. Buras, A. Poschenrieder, C. Tarantino, S. Uhlig and A. Weiler, Particle-Antiparticle Mixing, $\varepsilon_{K}, \Delta \Gamma_{q}, A_{\mathrm{SL}}^{q}, A_{\mathrm{CP}}\left(B_{d} \rightarrow \psi K_{S}\right), A_{\mathrm{CP}}\left(B_{s} \rightarrow \psi \phi\right)$ and $B \rightarrow X_{s, d} \gamma$ in the Littlest Higgs Model with T Parity, JHEP 12 (2006) 003 [hep-ph/0605214] [INSPIRE].

[61] M. Blanke, A.J. Buras, A. Poschenrieder et al., Rare and CP-Violating $K$ and $B$ Decays in the Littlest Higgs Model with $T^{-}$Parity, JHEP 01 (2007) 066 [hep-ph/0610298] [INSPIRE].

[62] M. Blanke, A.J. Buras and S. Recksiegel, Quark flavour observables in the Littlest Higgs model with T parity after LHC Run 1, Eur. Phys. J. C 76 (2016) 182 [arXiv:1507.06316] [INSPIRE].

[63] CMS, LHCB collaborations, Combination of results on the rare decays $B_{(s)}^{0} \rightarrow \mu^{+} \mu^{-}$from the CMS and LHCb experiments, CMS-PAS-BPH-13-007 (2014).

[64] CMS and LHCb Collaborations, Combination of results on the rare decays $B_{(s)}^{0} \rightarrow \mu^{+} \mu^{-}$ from the CMS and LHCb experiments, LHCb-CONF-2013-012 (2014).

[65] G. Bĺanger, F. Boudjema, A. Pukhov and A. Semenov, MicrOMEGAs4.1: two dark matter candidates, Comput. Phys. Commun. 192 (2015) 322 [arXiv:1407.6129] [INSPIRE].

[66] K. Griest and D. Seckel, Three exceptions in the calculation of relic abundances, Phys. Rev. D 43 (1991) 3191 [INSPIRE]. 
[67] D.S. Akerib et al., Results from a search for dark matter in the complete LUX exposure, arXiv: 1608.07648 [INSPIRE].

[68] PandaX-II collaboration, A. Tan et al., Dark Matter Results from First 98.7 Days of Data from the PandaX-II Experiment, Phys. Rev. Lett. 117 (2016) 121303 [arXiv:1607.07400] [INSPIRE].

[69] XENON collaboration, E. Aprile et al., Physics reach of the XENON1T dark matter experiment, JCAP 04 (2016) 027 [arXiv: 1512.07501] [INSPIRE].

[70] C. Han, A. Kobakhidze, N. Liu, A. Saavedra, L. Wu and J.M. Yang, Probing Light Higgsinos in Natural SUSY from Monojet Signals at the LHC, JHEP 02 (2014) 049 [arXiv: 1310.4274] [INSPIRE].

[71] C. Han, L. Wu, J.M. Yang, M. Zhang and Y. Zhang, New approach for detecting a compressed bino/wino at the LHC, Phys. Rev. D 91 (2015) 055030 [arXiv:1409.4533] [INSPIRE].

[72] K.-i. Hikasa, J. Li, L. Wu and J.M. Yang, Single top squark production as a probe of natural supersymmetry at the LHC, Phys. Rev. D 93 (2016) 035003 [arXiv:1505.06006] [INSPIRE].

[73] ATLAS collaboration, Search for new phenomena in final states with an energetic jet and large missing transverse momentum in pp collisions at $\sqrt{s}=8 \mathrm{TeV}$ with the ATLAS detector, Eur. Phys. J. C 75 (2015) 299 [Erratum ibid. C 75 (2015) 408] [arXiv:1502.01518] [INSPIRE].

[74] M. Drees, H. Dreiner, D. Schmeier, J. Tattersall and J.S. Kim, CheckMATE: Confronting your Favourite New Physics Model with LHC Data, Comput. Phys. Commun. 187 (2015) 227 [arXiv: 1312.2591] [INSPIRE].

[75] J.S. Kim, D. Schmeier, J. Tattersall and K. Rolbiecki, A framework to create customised LHC analyses within CheckMATE, Comput. Phys. Commun. 196 (2015) 535 [arXiv: 1503.01123] [INSPIRE].

[76] J. Alwall, R. Frederix, S. Frixione et al., The automated computation of tree-level and next-to-leading order differential cross sections and their matching to parton shower simulations, JHEP 07 (2014) 079 [arXiv: 1405.0301] [INSPIRE].

[77] T. Sjöstrand, S. Mrenna and P.Z. Skands, PYTHIA 6.4 Physics and Manual, JHEP 05 (2006) 026 [hep-ph/0603175] [INSPIRE].

[78] DELPHES 3 collaboration, J. de Favereau et al., DELPHES 3, A modular framework for fast simulation of a generic collider experiment, JHEP 02 (2014) 057 [arXiv:1307.6346] [INSPIRE].

[79] M. Cacciari, G.P. Salam and G. Soyez, FastJet User Manual, Eur. Phys. J. C 72 (2012) 1896 [arXiv: 1111.6097] [INSPIRE].

[80] M. Cacciari, G.P. Salam and G. Soyez, The Anti-kt jet clustering algorithm, JHEP 04 (2008) 063 [arXiv:0802.1189] [INSPIRE].

[81] Y. Xiao-Dong, X. Shou-Jian, M. Wen-Gan, Z. Ren-You, G. Lei and L. Xiao-Zhou, Precise predictions for $A_{H} q_{-}$associated production in the littlest Higgs model with $T$ parity at the LHC, Phys. Rev. D 89 (2014) 014008 [arXiv: 1312.1458] [INSPIRE]. 